\title{
On the invisible components of some eclipsing binaries
}

\author{
T. Borkovits and T. Hegedüs \\ Baja Astronomical Observatory of Bács-Kiskun County, Szegedi út, P.O. Box.766., H-6500, Baja, Hungary
}

Received October 30, 1995; accepted March 17, 1996

\begin{abstract}
The aim of this paper is to search for evidences for the presence of unseen companions in some eclipsing binary systems using their O-C diagrams. A total of 8507 times of minima (6890 visual and 1617 photographic or photoelectric ones) of 18 systems have been collected from the remarkable database of late Dieter Lichtenknecker (Lichtenknecker 1988) and from other more recent papers. Assuming that light-time effect (LITE) is responsible for the periodic components on the O-C diagrams sets of orbital parameters were derived using Kopal's (1978) method. In some cases (AB And, TV Cas, XX Cep, AK Her) the resulting orbital parameters and masses of the hypothetical third components are physically acceptable. Other systems show good evidence for multiplicity, but the available data sets are not suited to compute reliable solutions (W Del, U Peg, AT Peg, ST Per). For RT And, XZ And, OO Aql, Y Cam, RS CVn, CQ Cep, U CrB and MR Cyg) an unambigous identification of third components is not possible. In the cases of TW Cas and SW Lac we could not find any LITE solution at all.
\end{abstract}

Key words: binaries: eclipsing — binaries: close

\section{Introduction}

The problem of the existence of third (or further) components in eclipsing binary systems - either from a theoretical or observational point of view - has a long history, and quite extended literature exists. Three well-known basic studies are by Frieboes-Conde \& Herczeg (1973) and more recently by Mayer (1990) and Chambliss (1992). Further references can be found in these papers. Eclipsing binary systems provide a good opportunity for studying the presence of an unresolved third body by observing their times of minima because of the light-time effect (LITE), caused by the orbital motion of the eclipsing pair around the common centre of mass of the triple system. LITE usually results in a periodic component of the $(\mathrm{O}-\mathrm{C}) \mathrm{di}-$ agram with both primary and secondary minima running in the same phase, while apsidal motion (AM) which may also be responsible for periodic period variations cause a shift of the secondary minimum in opposite phase with respect to the primary minimum. Typically, the periods of known LITE range from 10 to 100 years, while AM periods usually are in the range from 100 to 2-3000 year. Therefore it is more probable that a short-term periodic component of an O-C is caused by LITE than AM, although there are cases where both effects are combined (e.g. RU Mon, U Oph). Unfortunately, the secondary minima are quite shallow in a large number of eclipsing systems (especially in Algol-type binaries) and observations

Send offprint requests to: T. Borkovits are often inadequate to distinguish between the two possibilities. Moreover, there are a number of mechanisms (magnetic cycle induced structural changes, mass transfer, internal motions caused by evolutionary effects, etc.), which can also cause detectable period variations, even cyclic period changes (magnetic cycle, e.g. Applegate \& Patterson 1987). Therefore the O-C diagram is usually a combination of several effects.

The purpose of this paper is to study the period variations of those eclipsing variable stars where there is an indication of any cyclic (periodic) behavior of the $\mathrm{O}-\mathrm{C}$ diagrams. In each case the working hypothesis was that the periodic term is the result of LITE caused by one or more invisible bodies. Thus, the system can be classified based on the results of the orbital analysis, i.e. it is possible to decide whether LITE can be used as an explanation of the periodic period variation or it requires other mechanisms.

The ideal case (when independent astrometric and spectroscopic measurements are available in addition to photometric data) occurs rarely (e.g. Algol, VW Cep, EE Peg). This is a consequence of the following selection effect: The amplitude of the $\mathrm{O}-\mathrm{C}$ curve for a well observable LITE must be at least 0.01 days. The radial velocity amplitude of the binary in the triple system (in the case of a circular orbit lying in the line of sight) is

$$
K_{12}=\frac{G^{1 / 2}}{A^{1 / 2} c^{1 / 2}} \frac{m_{3}^{3 / 2}}{m_{12}+m_{3}}
$$


where $G$ is the gravitational constant, $A$ is the amplitude of the $\mathrm{O}-\mathrm{C}$ curve, $c$ is the speed of light, $m_{12}$ is the total mass of the eclipsing pair, and $m_{3}$ is the mass of the third body. Choosing the most advantageous case with respect to the spectroscopic detection (relatively large third mass, small LITE-amplitude), say $m_{12}=2 M_{\odot}, m_{3}=0.5 M_{\odot}$ and $A=0$ d 01 , then Eq. (1) results in:

$$
K_{12}=2.28 \mathrm{~km} / \mathrm{s}
$$

which is close to the limit of detectability (see also Mayor \& Mazeh 1987). (The corresponding orbital period is 22.5 years.)

The photometrically detected third, fourth, etc. invisible satellites of eclipsing (or only spectroscopic) binaries should be studied with infrared CCDs, speckle interferometric techniques etc. for obtaining independent evidence of their existence.

\section{Observational material}

The majority of our data was obtained from the remarkable collection of the Belgian amateur astronomer Lichtenknecker (1988). We had to collect only the most recent observational reports for latest times of minima. The basic parameters of our program stars are summarized in Table 1. We used both visual (VIS) and photographic/photoelectric (PGPE) observations (the relative percentage of the latter being one-fourth of all the observations), in spite of the fact that earlier visual data have relatively large errors. Disregarding these, however, would result in a total loss of information when the system was observed only visually (especially at early epochs). Therefore, they were taken into account, but with a 1/10 weight relative to the photoelectric data. The weights used were the following: visual $=1$, photographic (plate minimum $)=2$, photographic (seasonal light curve $)=5$, photoelectric $=10$. The estimated errors of these minima are 0.01 day for visual and 0.001 day for photoelectric data.

In our cases, the examined $\mathrm{O}-\mathrm{C}$ diagrams had well discernible periodic behaviour with amplitudes of several hundreds of a day. Thus, this was another reason why we kept the visual results in some cases, even if only for a first approximation. We present the raw $\mathrm{O}-\mathrm{C}$ diagrams of all 18 stars in Figs. 1a-r. The linear ephemeris was taken from GCVS 4th edition (Kholopov et al. 1985) for the construction of these diagrams.

Table 1 does not contain all the references to the enormous amount of times of minima. All these data, however, can be requested either in electronic or printed form from the authors. We shall send the ordered data in printed form (as a xeroxcopy of the original printing of Lichtenknecker's tables, with exact references), or as an ASCII file (via E-mail or on IBM PC formatted diskette; without reference list).

\section{Analysis}

The analysis of all data sets was performed in the following way. As a first step, in some cases it was necessary to remove a second order term (parabola) from the $\mathrm{O}-\mathrm{C}$ diagram. Secondly, the Fourier spectra of all the $\mathrm{O}-\mathrm{C}$ data were computed using the Discrete Fourier Transformation method, and approximate frequency harmonics were identified. These frequencies were refined with weighted least-squares fitting. Finally, the orbital parameters were derived based on the following formulae (Kopal 1978):

$$
\begin{gathered}
A_{1}^{\prime} \sin i^{\prime}=c \sqrt{a_{1}^{2}+b_{1}^{2}}, \\
e^{\prime}=2 \sqrt{\frac{a_{2}^{2}+b_{2}^{2}}{a_{1}^{2}+b_{1}^{2}}}, \\
\omega^{\prime}=\arctan \frac{\left(b_{1}^{2}-a_{1}^{2}\right) b_{2}+2 a_{1} a_{2} b_{1}}{\left(a_{1}^{2}-b_{1}^{2}\right) a_{2}+2 a_{1} b_{1} b_{2}}, \\
\tau^{\prime}=t_{0}-\frac{P^{\prime}}{2 \pi} \arctan \frac{a_{1} b_{2}-b_{1} a_{2}}{a_{1} a_{2}+b_{1} b_{2}},
\end{gathered}
$$

where $a_{1,2}, b_{1,2}$ are the Fourier coefficients coming from the analysis as described above (i.e. the method needs first harmonics of the fundamental frequency), $A^{\prime}$ denotes the semi-major axis of the absolute orbit of the centre of mass of the eclipsing pair around that of the triple system, $i^{\prime}$, $e^{\prime}, \omega^{\prime}, t^{\prime}$ and $P^{\prime}$ are the usual elements of the third body orbit. $P$ is the sidereal period of the close pair, while $c$ is the speed of light.

These equations can be considered to be a good approximation for smaller eccentricities. During the test of our procedure we could obtain reliable results for $e^{\prime}<0.6$. Thus, for several cases we used only the first-order approximation shown above.

Usually, the above method was applied twice: first, all times of minima (TOT) were analyzed, then it was restricted to the PGPE data. There was one case (MR Cyg) where it was necessary to use only the photoelectric observations for achieving useful results.

When the quality of the observational material and the behaviour of the observed variation was adequate, we used the second-order approximation for determination of orbital data. By this method one can determine the orbital elements as follows:

$$
\begin{gathered}
e^{\prime}=\frac{4}{3} \sqrt{\frac{a_{3}^{2}+b_{3}^{2}}{a_{2}^{2}+b_{2}^{2}}}, \\
\omega^{\prime}=\arctan \left(\frac{\left(b_{1}^{2}-a_{1}^{2}\right) b_{2}+2 a_{1} a_{2} b_{1}}{\left(a_{1}^{2}-b_{1}^{2}\right) a_{2}+2 a_{1} b_{1} b_{2}}\left(1-\frac{e^{2}}{3}\right)\right), \\
\tau^{\prime}=t_{0}-\frac{P^{\prime}}{2 \pi} \arctan \frac{1-\frac{a_{1}}{b_{1}} \frac{h_{1}}{g_{1}} \tan \omega^{\prime}}{\frac{a_{1}}{b_{1}}+\frac{h_{1}}{g_{1}} \tan \omega^{\prime}}, \\
A_{1}^{\prime} \sin i^{\prime}=c \frac{a_{1}^{2}+b_{1}^{2}}{h_{1}^{2}+\left(g_{1}^{2}-h_{1}^{2}\right) \cos \omega^{\prime 2}},
\end{gathered}
$$


Table 1. Basic data for the systems involved in this study. $\mathrm{T}$ is the type of the light changes (A: Algol, W: W UMa, $\beta$ : $\beta$ Lyr), TOT means the total number of all accepted times of minima of the stars, PGPE is the number of the photographic and photoelectric times of minima, mag is the brightness of the system in maximum and minimum light (p: photographic, V: $V$-filter photoelectric stellar magnitudes), $s p_{1}+s p_{2}$ gives the spectral types of the components according to GCVS, $M_{1}, M_{2}$ are the masses of the components in solar masses and $P$ is the approximate period of the light changes (in days)

\begin{tabular}{|c|c|c|c|c|c|c|c|c|c|c|}
\hline \multirow[t]{2}{*}{ Name } & \multirow[t]{2}{*}{$\mathrm{T}$} & \multirow[t]{2}{*}{ TOT } & \multirow[t]{2}{*}{ PGPE } & \multicolumn{2}{|c|}{ mag } & \multirow[t]{2}{*}{$s p_{1}+s p_{2}$} & \multirow[t]{2}{*}{$M_{1}$} & \multirow[t]{2}{*}{$M_{2}$} & \multirow[t]{2}{*}{$P$} & \multirow[t]{2}{*}{ Sources } \\
\hline & & & & $\max$ & $\min$ & & & & & \\
\hline RT And & A & 729 & 97 & 8.55 & $9.47 \mathrm{~V}$ & F8V & 1.52 & 1.00 & 0.63 & 1,2 \\
\hline XZ And & A & 753 & 75 & 10.02 & $12.99 \mathrm{p}$ & A0+G8:III. & 3.23 & 1.65 & 1.36 & 1,3 \\
\hline AB And & $\mathrm{W}$ & 1148 & 187 & 9.50 & $10.32 \mathrm{~V}$ & G5+G5V & 1.06 & 1.71 & 0.33 & 1,4 \\
\hline OO Aql & $\mathrm{W}$ & 1085 & 77 & 9.2 & $9.9 \mathrm{~V}$ & G5V & \multicolumn{2}{|c|}{$\Sigma=2.5$} & 0.51 & 1,8 \\
\hline Y Cam & $\mathrm{A}$ & 248 & 57 & 10.50 & $12.24 \mathrm{~V}$ & $\mathrm{~A} 8 \mathrm{~V}$ & 2.33 & 0.50 & 3.31 & 1,2 \\
\hline RS CVn & $\mathrm{A}$ & 93 & 58 & 7.93 & $9.14 \mathrm{~V}$ & $\mathrm{~F} 4 \mathrm{IV}+\mathrm{K} 0 \mathrm{IVe}$ & 1.42 & 1.35 & 4.80 & 1,2 \\
\hline TV Cas & A & 657 & 77 & 7.22 & $8.22 \mathrm{~V}$ & B9V+F7IV & 4.04 & 1.62 & 1.81 & 1,5 \\
\hline TW Cas & A & 168 & 72 & 8.32 & $8.98 \mathrm{~V}$ & $\mathrm{~B} 9 \mathrm{~V}+\mathrm{A} 0$ & 2.90 & 1.18 & 1.43 & 1,2 \\
\hline XX Cep & A & 191 & 59 & 9.13 & $10.28 \mathrm{p}$ & $\mathrm{A} 8 \mathrm{~V}$ & 1.87 & 0.32 & 2.34 & 1,5 \\
\hline CQ Cep & $\beta$ & 58 & 38 & 8.63 & $9.12 \mathrm{~V}$ & WN5.5+O7 & 17.5 & 21.1 & 1.64 & 1,6 \\
\hline $\mathrm{U}$ CrB & A & 269 & 40 & 7.66 & $8.79 \mathrm{~V}$ & $\mathrm{~B} 6 \mathrm{~V}+\mathrm{F} 8 \mathrm{III}$ & 4.7 & 4.4 & 3.45 & 1,11 \\
\hline MR Cyg & A & 120 & 53 & 8.75 & $9.68 \mathrm{~V}$ & $\mathrm{~B} 3 \mathrm{~V}+\mathrm{B} 9$ & 7.6 & 5.7 & 1.68 & 1,9 \\
\hline W Del & A & 184 & 32 & 9.69 & $12.33 \mathrm{~V}$ & $\mathrm{~B} 9.5 \mathrm{Ve}+\mathrm{G} 5$ & 2.01 & 0.42 & 4.81 & 1,5 \\
\hline AK Her & $\mathrm{W}$ & 330 & 177 & 8.29 & $8.77 \mathrm{~V}$ & $\mathrm{~F} 2+\mathrm{F} 6$ & \multicolumn{2}{|c|}{$\Sigma=1.5$} & 0.42 & 1,7 \\
\hline SW Lac & $\mathrm{A}$ & 1669 & 402 & 8.51 & $9.39 \mathrm{~V}$ & $\mathrm{G} 8 \mathrm{Vp}+\mathrm{G} 8 \mathrm{Vp}$ & 0.96 & 1.14 & 0.32 & 1,4 \\
\hline U Peg & A & 423 & 79 & 9.23 & $10.07 \mathrm{~V}$ & $\mathrm{~F} 3+\mathrm{F} 3$ & 1.29 & 0.86 & 0.37 & 1,2 \\
\hline AT Peg & $\mathrm{A}$ & 176 & 26 & 8.97 & $9.75 \mathrm{~V}$ & $\mathrm{~A} 7 \mathrm{~V}$ & 2.2 & 0.93 & 1.15 & 1,10 \\
\hline ST Per & $\mathrm{A}$ & 206 & 11 & 9.52 & $11.40 \mathrm{~V}$ & $\mathrm{~A} 3 \mathrm{~V}+\mathrm{G}-\mathrm{K}$ & 2.03 & 0.39 & 2.65 & 1,2 \\
\hline
\end{tabular}

Sources: 1) Kholopov et al. 1985; 2) Giannone \& Giannuzzi 1974; 3) Budding 1984; 4) Rovithis-Livaniou et al. 1990; 5) Van Hamme \& Wilson 1990; 6) Kartasheva \& Svechnikov 1986; 7) Nagy 1985; 8) Hrivnak 1989; 9) Linnell \& Kallrath 1987; 10) Hill \& Barnes 1972; 11) Heintze 1990.

where

$$
g_{1}=1-\frac{5 e^{2}}{8}
$$

$$
\begin{aligned}
m_{3}^{3} \sin ^{3} i^{\prime}-m_{3}^{2} f\left(m_{3}\right) & -2 m_{3} m_{12} f\left(m_{3}\right) \\
& -m_{12}^{2} f\left(m_{3}\right)=0
\end{aligned}
$$

and

$$
h_{1}=1-\frac{3 e^{\prime 2}}{8}
$$

(Vinkó 1989). Other designations are the same as above. Because of the features of the Fourier-analysis, it was sometimes necessary to add $P^{\prime} / 2$ to the value of $t^{\prime}$. Of course, this approximation needs two harmonics of the fundamental frequency. Respective coefficients are $a_{1,2,3}$ and $b_{1,2,3}$.

Having the orbital parameters determined in either way, the mass function of the companion can be expressed as follows:

$$
f\left(m_{3}\right)=\frac{m_{3}^{3} \sin ^{3} i^{\prime}}{\left(m_{12}+m_{3}\right)^{2}}=\frac{4 \pi^{2} A_{1}^{\prime 3} \sin ^{3} i^{\prime}}{G P^{\prime 2}}
$$

where $m_{12}$ and $m_{3}$ are the mass of the eclipsing pair and the third body, respectively, and $G$ is the gravitational constant. The unknown mass $m_{3}$ can be derived from a third-order equation:

Of course, the result will contain a free parameter $\left(\sin i^{\prime}\right)$ which remains undeterminable from these kinds of observations. We shall present (as it was done usually by other authors) the masses of the hypothetical satellites for a few different orbital inclinations.

\section{Results}

\subsection{Group I. (Likely Cases)}

\subsubsection{AB And}

The eclipsing binary nature of this variable star was discovered by Guthnick and Prager in 1927. Although AB And was mentioned among the systems having O-C curves which are superposition of a parabola and a sinusoidal curve (Kreiner 1971), later it was stated to be a system with constant period (Panchatsaram \& Abhyankar 1982a).

The $\mathrm{O}-\mathrm{C}$ diagram based on the linear ephemeris in GCVS 4th ed. (Kholopov et al. 1985) can be seen in Fig. 1c. After removing a least-squares parabola one can 

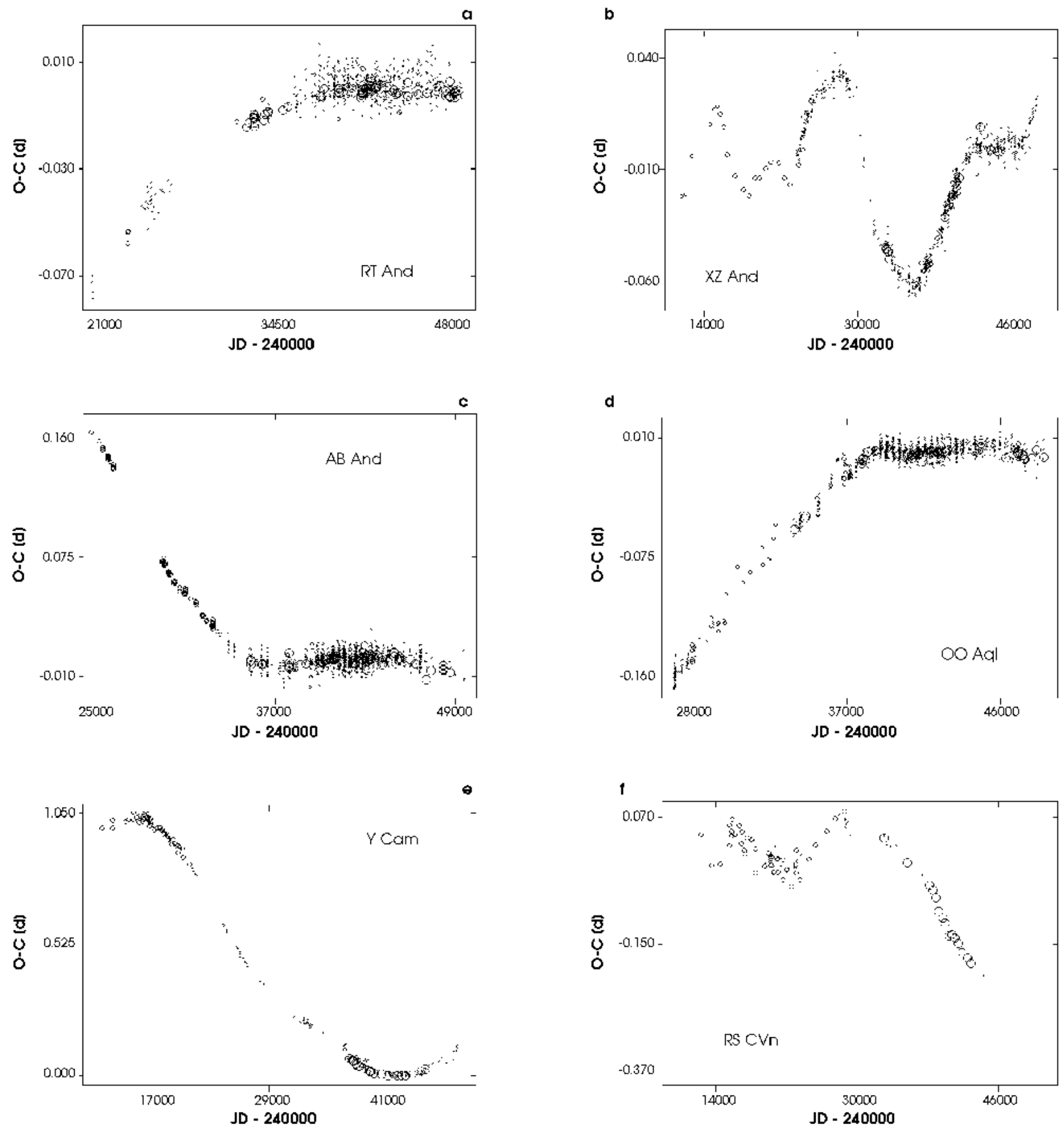

Fig. 1. a-f) Raw O-C diagrams based on GCVS (1985) ephemerides. The size of the circles is proportional to the statistical weights assigned to the individual minima (visual - 1; plate-minima - 2; photographic - 5; photoelectric measurement - 10) 

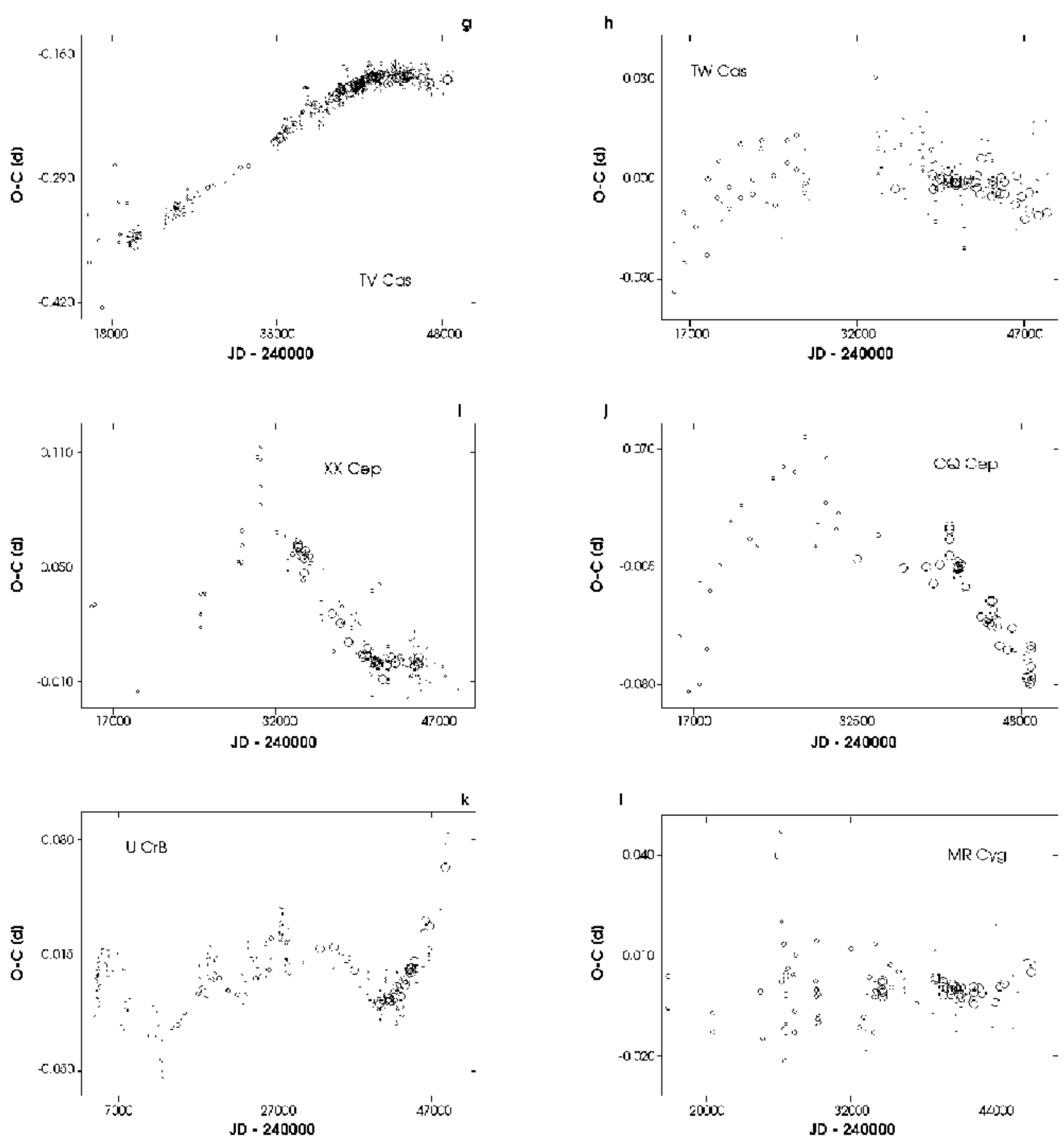

Fig. 1. g-l) Raw O-C diagrams with the GCVS (1985) ephemerides

obtain a reasonable LITE fit to the residuals which is shown in Fig. 2 (the quadratic ephemeris is listed in Table 2). The orbital elements derived are collected in Table 3. Note that due to the smaller eccentricity the firstorder method was used and some uncertain points outside the $3 \sigma$ limit were removed. The final fit revealed a small-mass third body revolving in an almost circular orbit. Supposing a main-sequence satellite, it could be for example an M0V dwarf (for $i^{\prime}=60^{\circ}$, which is $4 \mathrm{mag}$ fainter than the binary itself). (We identified the spectral types of the third components and calculated the 

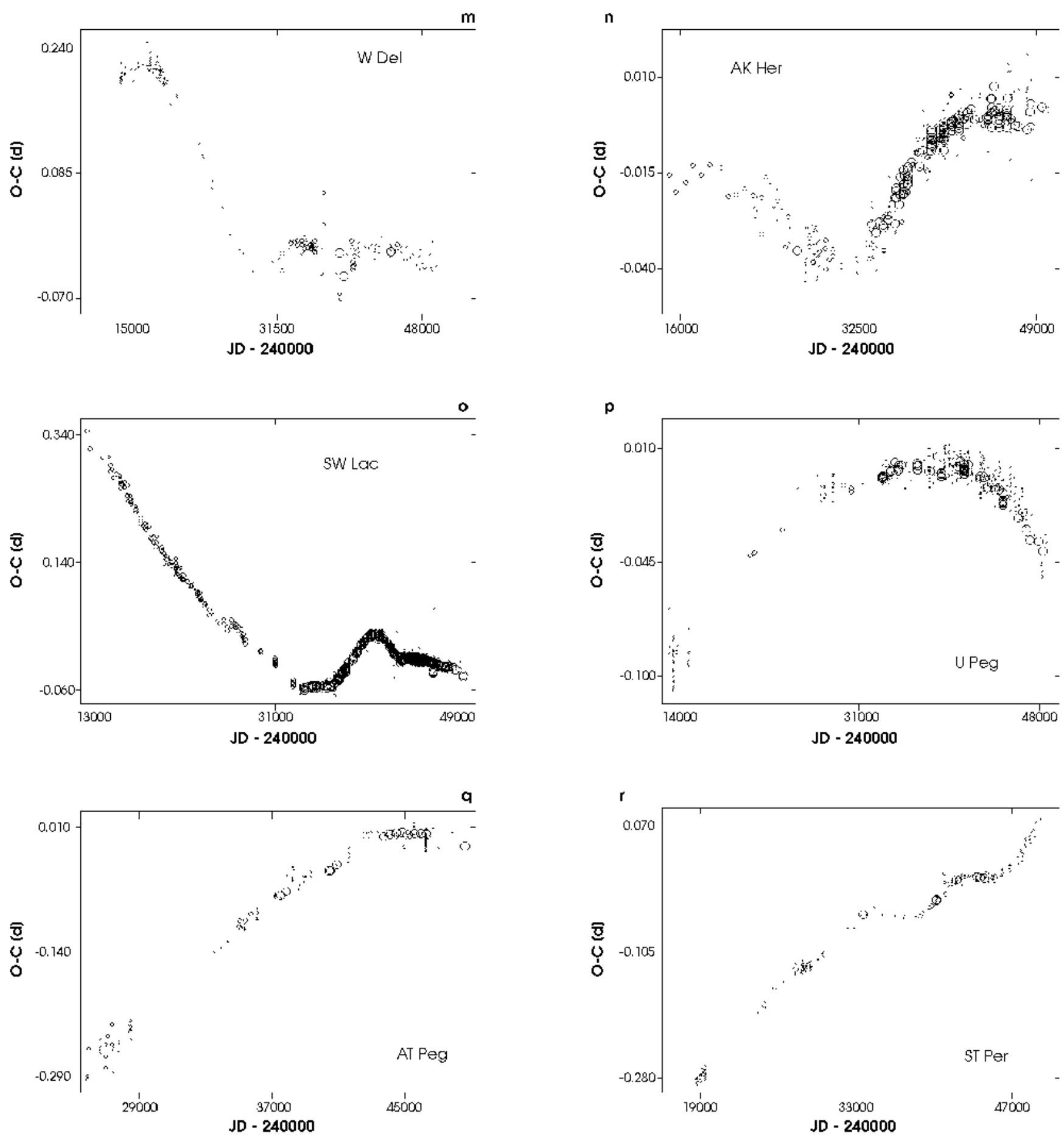

Fig. 1. m-r) Raw O-C diagrams with the GCVS (1985) ephemerides

magnitude differences on the basis of Table 1.2. in de Loore \& Doom 1992).

\subsubsection{TV Cas}

The period variations of this Algol-type binary had different explanations. Plavec et al. (1961) have found a con- stant period, while Frieboes-Conde \& Herczeg (1973) revealed a cyclic variation in period which was thought to be the result of LITE or AM. Rafert (1982) derived a parabolic and a sinusoidal ephemeris with very small amplitude which would have needed alternative explanation. Panchatsaram \& Abhyankar (1982a, b) found a secular 
Table 2. Ephemerides used for the $\mathrm{O}-\mathrm{C}$ diagrams

\begin{tabular}{llllll}
\hline AB And & Min I pgpe: & 2436109.58041 & $+0.33188985 E$ & $+5.33510^{-11} E^{2}$ & present paper \\
TV Cas & Min I pgpe: & 2444662.27198 & $+1.81260 E$ & $-8.69710^{-10} E^{2}$ & present paper \\
XX Cep & Min I pgpe: & 2444839.8022 & $+2.33732665 E$ & & GCVS 1985 \\
AK Her & Min I pgpe: & 2442186.460 & $+0.42152227 E$ & & Barker\&Herczeg (1979) \\
U CrB & Min I tot: & 2437844.37911 & $+3.45220552 E$ & & Mayer et al. (1991) \\
W Del & Min I tot: & 2443328.52755 & $+4.80610015 E$ & $+7.25310^{-9} E^{2}$ & present paper \\
U Peg & Min I pgpe: & 2436511.66821 & $+0.374781439 E$ & & GCVS 1985 \\
AT Peg & Min I pgpe: & 2445219.85614 & $+1.1460796 E$ & $-1.0510^{-9} E^{2}$ & present paper \\
ST Per & Min I tot: & 2442436.5779 & $+2.6483418 E$ & & present paper \\
RT And & Min I pgpe: & 2441141.88901 & $+0.628929513 E$ & & GCVS 1985 \\
XZ And & Min I tot: & 2423977.1915 & $+1.357278 E$ & & GCVS 1985 \\
OO Aql & Min I tot: & 2438613.21434 & $+0.5067914 E$ & $-1.61810^{-10} E^{2}$ & present paper \\
Y Cam & Min I tot: & 2442961.9276 & $+3.3056244 E$ & & GCVS 1985 \\
RS CVn & Min I pgpe: & 2422811.69133 & $+4.7978765 E$ & $-6.59310^{-9} E^{2}$ & present paper \\
TW Cas & Min I tot: & 2442008.3873 & $+1.4283240 E$ & & GCVS 1985 \\
CQ Cep & Min I tot: & 2432456.706 & $+1.641247 E$ & $-1.0510^{-9} E^{2}$ & present paper \\
MR Cyg & Min I pgpe: & 2433396.4096 & $+1.67703362 E$ & & GCVS 1985 \\
\hline
\end{tabular}

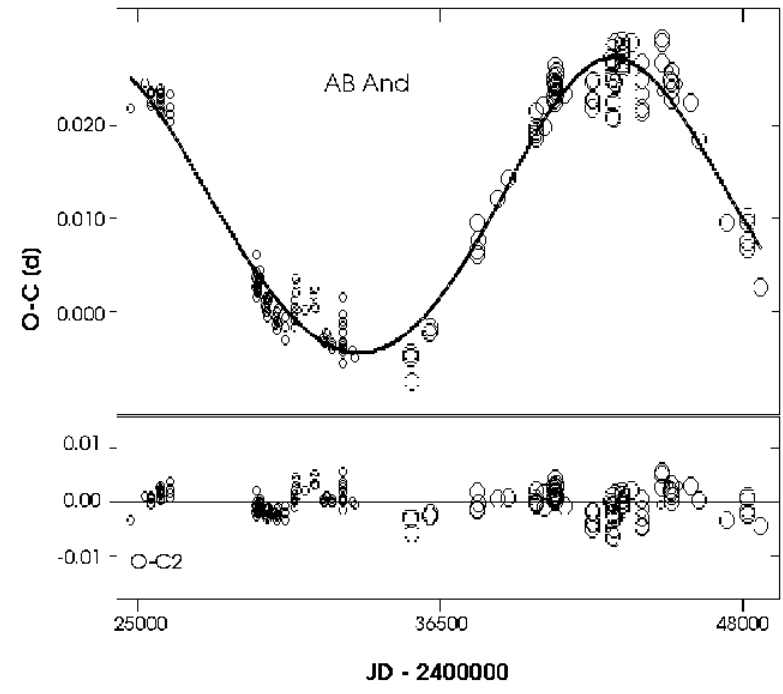

Fig. 2. LITE solution and residuals for $\mathrm{AB}$ And

period variation - although they could not exclude the possibility that the parabolic $\mathrm{O}-\mathrm{C}$ can be a part of a longer period sinusoid due to LITE of an invisible companion.

The original $\mathrm{O}-\mathrm{C}$ diagram is depicted in Fig. 1g. First, we have removed a parabola from the PGPE data (see Table 2). The residuals were analysed by the method described above. The resultant orbital parameters can be found in Table 3, while the theoretical fit and the original PGPE data are in Fig. 3. The procedure was the same as for $\mathrm{AB}$ And. Most probably, the hypothetical third body is an $\mathrm{M} 2 \mathrm{~V}$ red dwarf (for $i^{\prime}=60^{\circ}$ ) which is more than 9 mag fainter than the eclipsing system. The lack of observations of the secondary minimum prevents the unam- bigous identification of the periodic term as LITE, thus, further accurate observations are strongly needed.

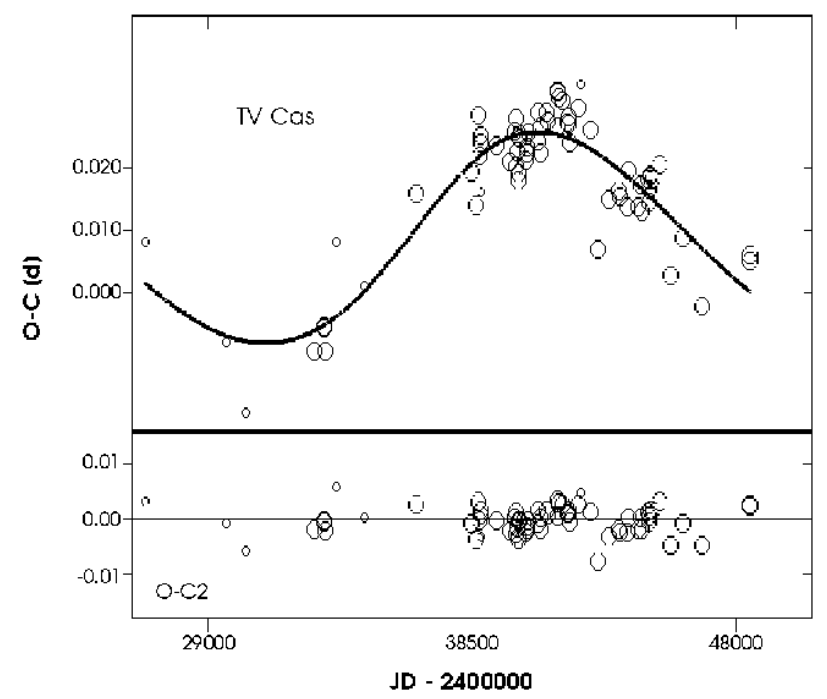

Fig. 3. LITE solution and residuals for TV Cas

\subsubsection{Cep}

This Algol-type eclipsing binary shows the effect of apsidal motion (Fresa 1956). Rafert (1982) identified a sudden period jump near J.D. 2425800 instead, and could not find the periodic term. It was, however, confirmed later by Mayer (1984) who determined its period as $P=64.2$ years and amplitude as $A=0.0371$.

Using the linear ephemeris of GCVS (Kholopov et al. 1985; Table 2) we have found an acceptable periodic change in the $\mathrm{O}-\mathrm{C}$ diagram. Results of the LITE solution 
can be found in Table 3, while the PGPE theoretical fit with the observational data is plotted in Fig. 4. This fit was achieved by using the original DFT period, but with a least-squares search for the best orbital parameters. The data outside the $3 \sigma$ limit were omitted at the final solution.

The mass function indicates a more massive component (cf. Mayer 1984) than in the previous cases. If it were not a white dwarf, it should be noticeable in the light curve as third light, and also in the combined spectrum (e.g. for $i^{\prime}=60^{\circ}: s p 3 \simeq G 1 V, \operatorname{mag}_{3}-\operatorname{mag}_{12} \simeq 2.4 \mathrm{mag}$ ). Moreover, AM cannot be excluded as an alternative explanation. The system is considered to be an apsidal motion candidate star in several studies (Kopal 1978; Hegedüs 1988). Unfortunately, there is a remarkable lack of exact timings of secondary minima due to the shallow eclipse (the dept of secondary minimum is about 0.16 mag in $\mathrm{V}$ ).

Thus, it would be necessary to measure the secondary minima of this system for making it clear which kind of effect is responsible for the observed behaviour of its $\mathrm{O}-\mathrm{C}$ diagram.

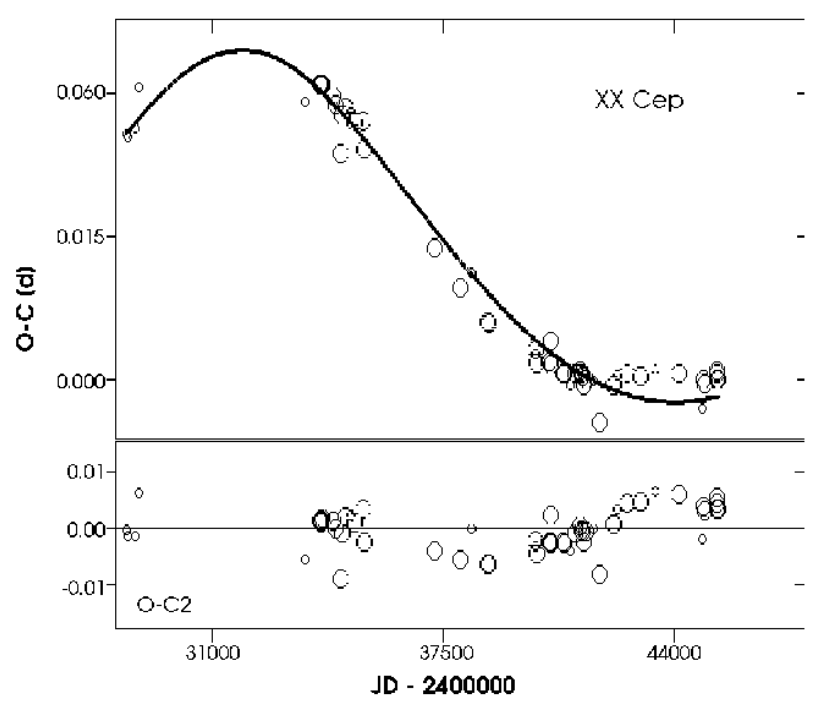

Fig. 4. LITE solution and residuals for XX Cep

\subsubsection{AK Her}

This W UMa type system has a visual companion at 4."7 distance, which corresponds to 450 A.U. (AK Her is at a distance of $95 \mathrm{pc}$ ). The orbital period is of the order of thousand years. Thus, it is clear that the visual companion cannot be responsible for the observed periodic changes of the $\mathrm{O}-\mathrm{C}$ diagram of AK Her. Different studies put the period of LITE between 56 and 78 years. Abhyankar \& Panchatsharam (1982) assumed two invisible, unresolved satellites of the eclipsing binary. We don't think that the observations are spread over such a long time interval that it would be necessary to postulate the presence of two companions instead of only one. Moreover minima times more recent than considered in the paper of Abhyankar \& Panchatsharam (1982) show a rather strange distribution. All data hardly cover more than one complete period. Thus, up to this moment it is very hard to make a firm statement on the existence of either one or two invisible companions.

One can see the raw $\mathrm{O}-\mathrm{C}$ diagram of $\mathrm{AK}$ Her in Fig. 1n. In the analysis we used the zero epoch published in GCVS 4th ed. (Kholopov et al. 1985) combined with the period of Barker \& Herczeg (1979). Within the limits of the probable errors, we have considered one LITE orbit to explain the observed behaviour of its $\mathrm{O}-\mathrm{C}$ diagram. The procedure was carried out only with PGPE data in the same manner as for the first two stars. The orbital elements can be seen in Table 3. The corresponding O-C diagram and the theoretical fit (whith the PGPE data) are shown in Fig. 5. The most uncertain parameter of this solution is the orbital eccentricity. Unfortunately, due to World War II., the minima times are missing just around the minimum of the diagram.

Probably, the third component may be a faint red dwarf. Woodward \& Wilson (1977) have not found noticeable evidence of third light in the light curve. If the third object was a main-sequence star it would be almost 7 mag fainter than the eclipsing pair (in the case of $i^{\prime}=60^{\circ}$ ).

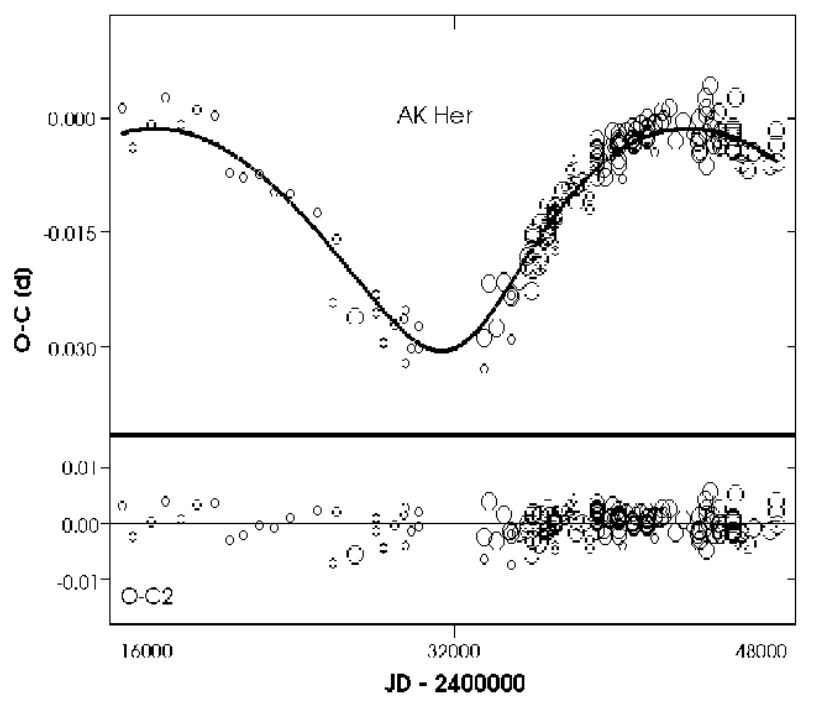

Fig. 5. LITE solution and residuals for AK Her

\subsection{Group II. (Problematic Cases)}

\subsubsection{W Del}

This Algol-like eclipsing variable was discovered by Ms Wells in 1895. Its period variation was recognized by Russell, Fowler \& Borton (1917). Plavec (1959) computed a linear and sinusoidal ephemeris. The 50.9 year-long period which he found could not be interpreted by the 


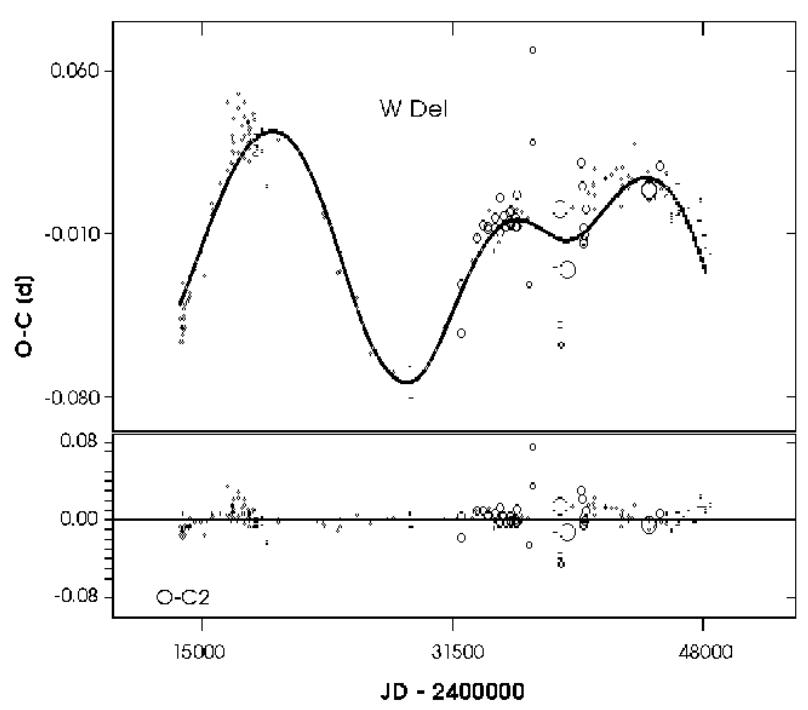

Fig. 6. A four body LITE solution and residuals for W Del

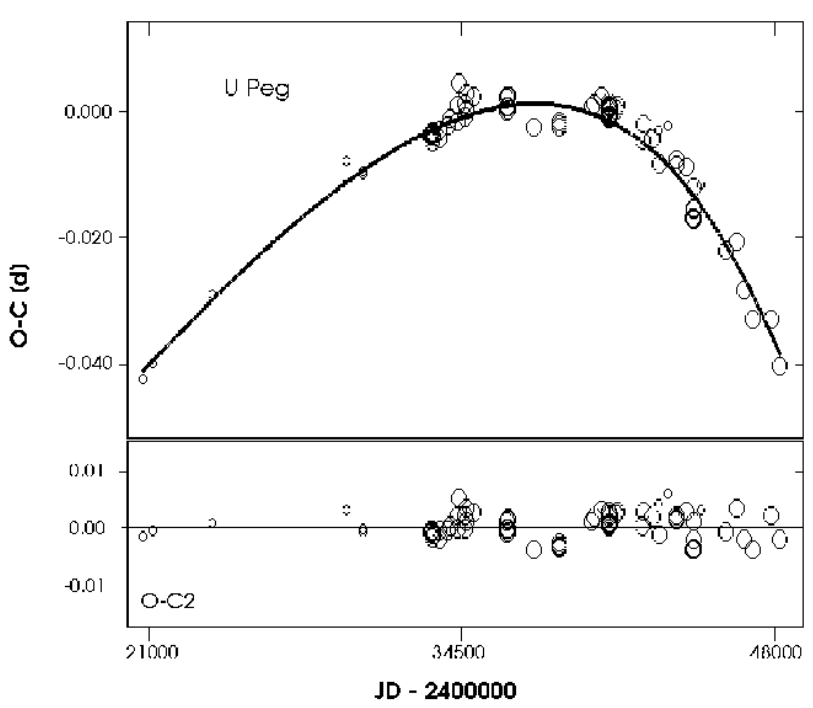

Fig. 7. LITE solution and residuals for U Peg

presence of either an invisible third component in the system or by AM. Nodal motion was excluded as well. Kreiner (1971) concluded that the star shows LITE due to one satellite.

The O-C curve (Fig. $1 \mathrm{~m}$ ) after removing the quadratic term (Table 2) could be described with two periodic components. During the analysis all data had to be used due to the very limited number of PGPE observations. The combined theoretical curve is plotted in Fig. 6 using the parameters collected in Table 4. Because of the inferior quality of the data used and the necessity of two companions (which have quite large minimal mass) in the LITE solution, the existence of perturbing bodies is questionable.

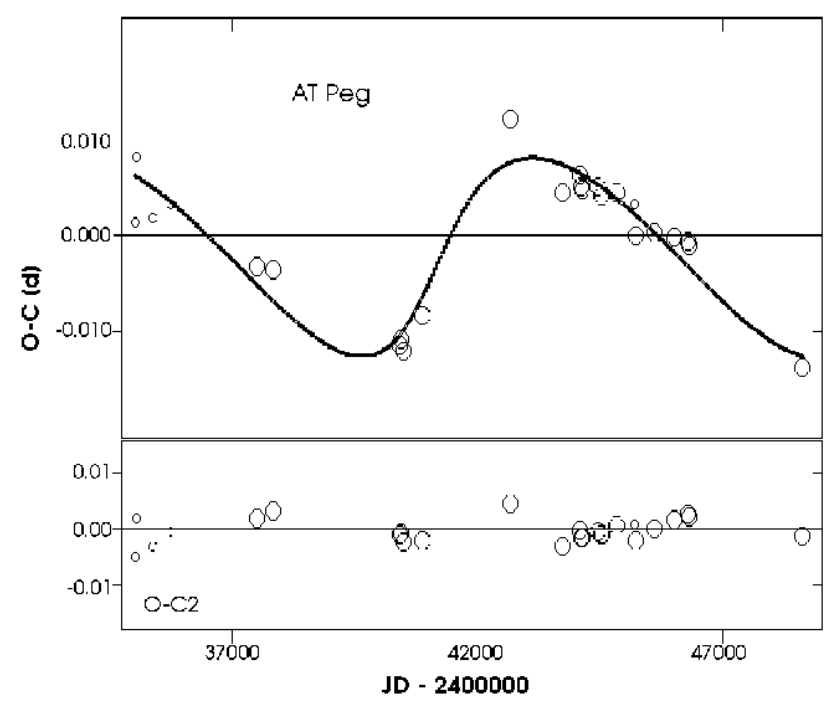

Fig. 8. LITE solution and residuals for AT Peg

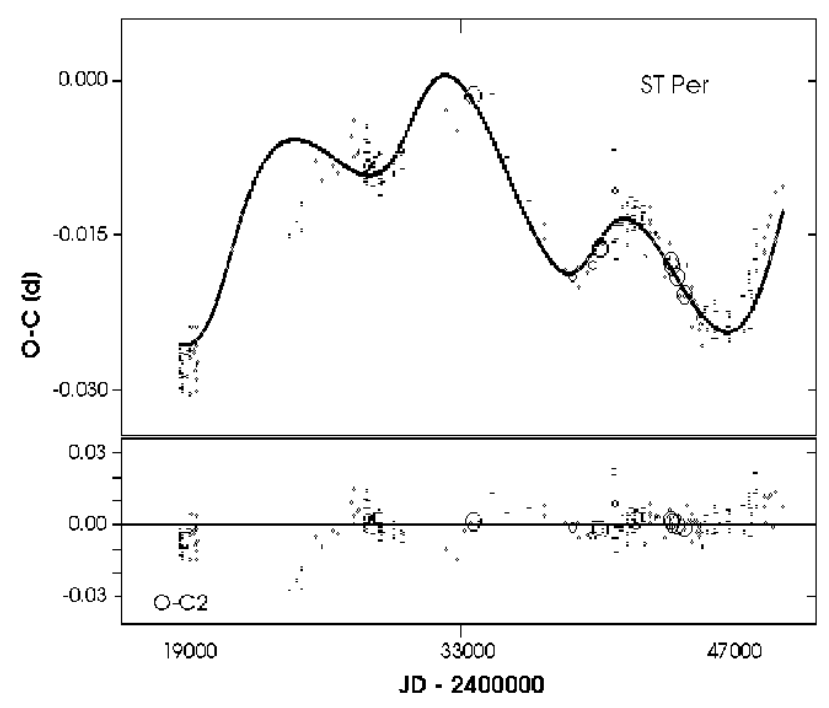

Fig. 9. A four body LITE solution and residuals for ST Per

\subsubsection{U Peg}

The period variation of this W UMa-type system (plotted in Fig. 1p) is often described as a secular one, i.e. the $\mathrm{O}-\mathrm{C}$ diagram is apparently parabolic (see e.g. Panchatsaram \& Abhyankar 1982b). The first step of our analysis was the removal of the following least-squares parabola:

$$
\operatorname{Min} I_{\text {TOT }}=2436511.671+0.37478 E-2.67310^{-11} E^{2}
$$

Due to the slight asymmetry of the ascending and descending branches of the observational data we assumed that in this case we can see a consequence of a LITE orbit with moderate eccentricity rather than an $\mathrm{O}-\mathrm{C}$ diagram of a secular period variation. Our PGPE 


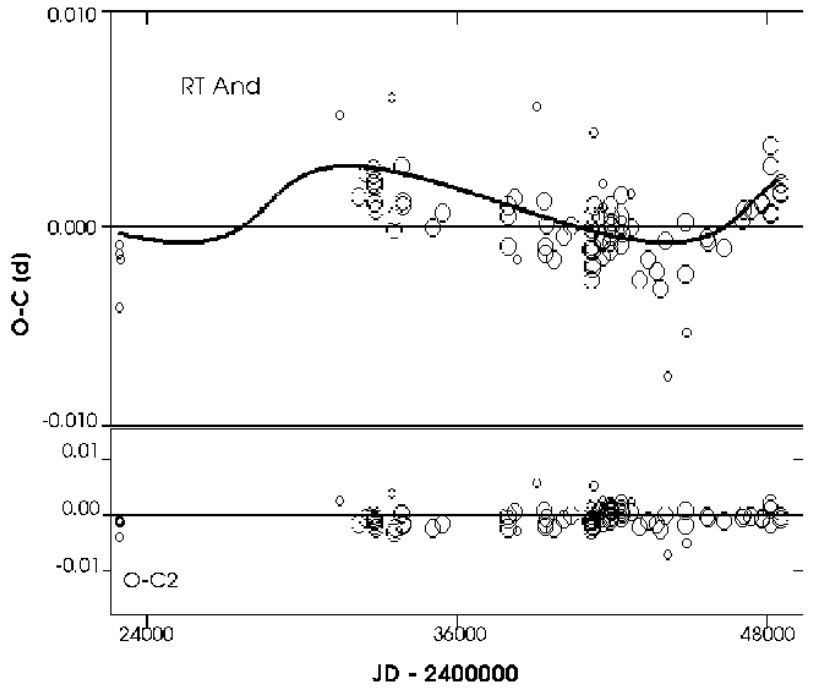

Fig. 10. PGPE points of RT And and the fourth body representation (after whitening with the third body effect)

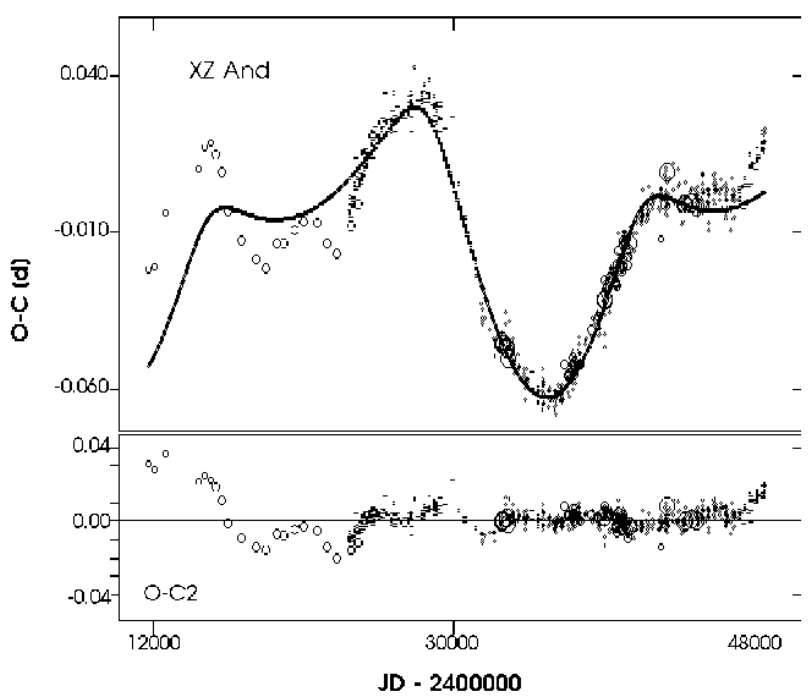

Fig. 11. TOT points of XZ And with our 'best' solution

results are strongly different from those of Panchatsaram \& Abhyankar (1982a), and can be seen in Fig. 7. The corresponding orbital elements are listed in Table 4. We put the system into Group II. because the observations cover less than a half period of the hypothetical third body which renders the solution less reliable. The third body may be a white dwarf.

\subsubsection{AT Peg}

This is a semi-detached Algol-like system. There are a few detailed investigations made earlier. We used the study of Hill \& Barnes (1972) for the spectroscopic elements. The raw $\mathrm{O}-\mathrm{C}$ can be seen in Fig. 1q. Removing a least-squares

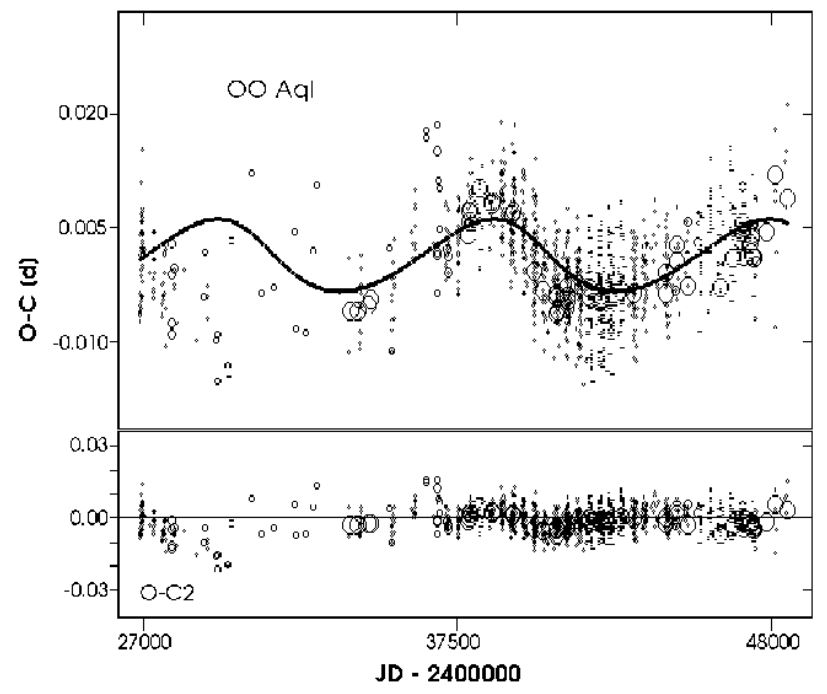

Fig. 12. TOT points of OO Aql whith LITE solution made after the removal of a parabola

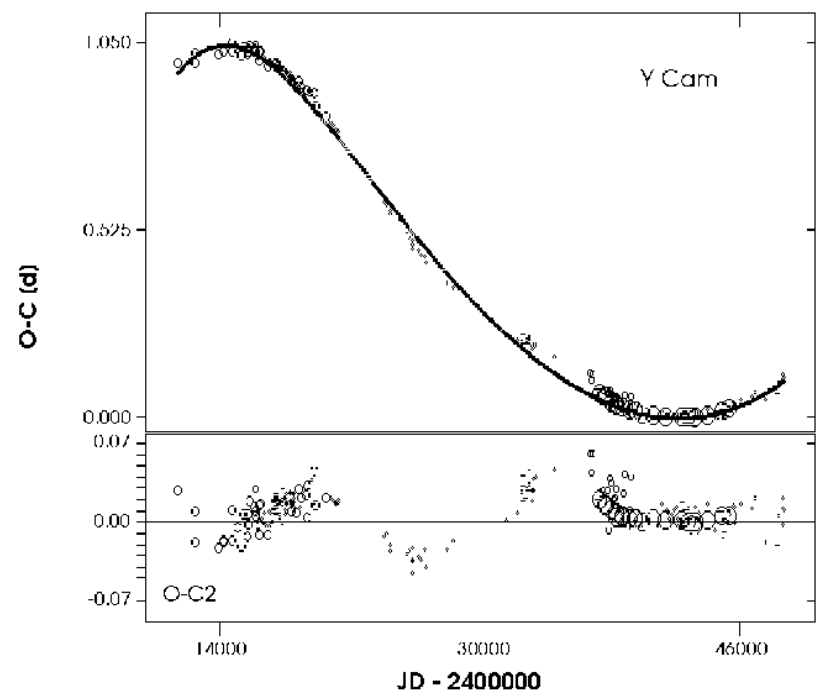

Fig. 13. TOT points of Y Cam with our formal LITE solution

parabola (Table 2), we could choose one main frequency from the DFT method. As usually, we improved its value by weighted least-squares fitting. Orbital parameters obtained by the second-order approximation are in Table 4, while the theoretical curve fitted to the PGPE data plotted in Fig. 8. The mass is very similar to the previous case, thus, this invisible component may be a white dwarf.

\subsubsection{ST Per}

The raw O-C diagram of this Algol-type system (Fig. 1r) indicates the necessity of improving the earlier ephemeris found in GCVS. The new ephemeris can be found in 


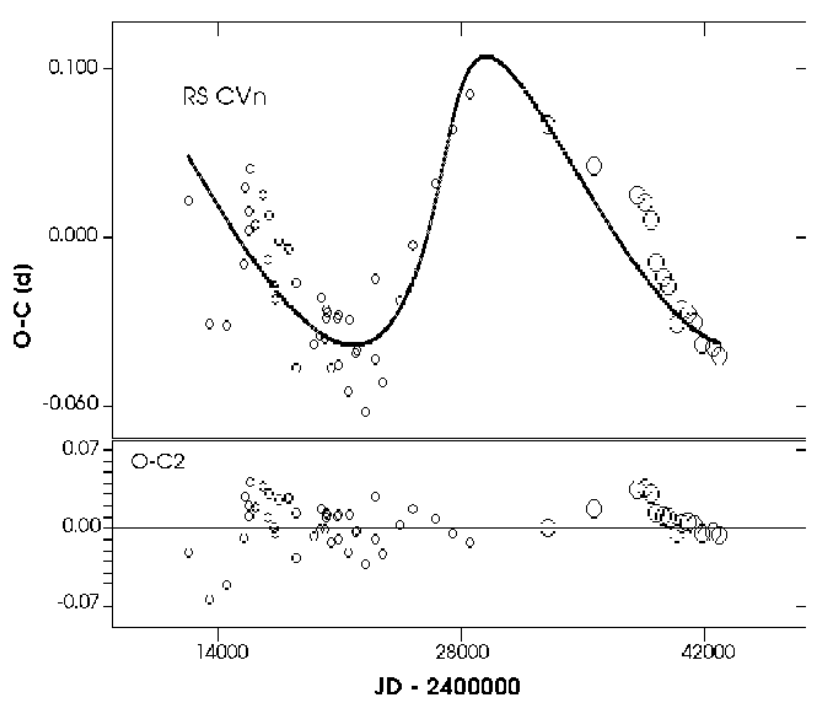

Fig. 14. PGPE points of RS CVn with LITE solution made after the removal of a parabola

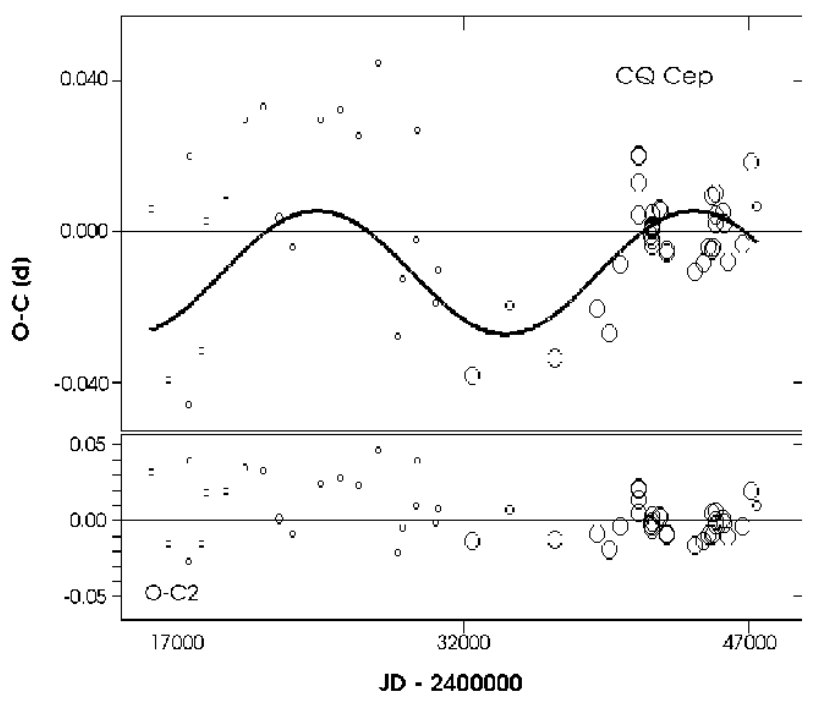

Fig. 15. TOT points of CQ Cep with LITE solution made after the removal of a parabola

Table 2. Analyzing the residuals two LITE solutions could be obtained using all data (Table 4) which are plotted in Fig. 9. The orbits of two perturbing components are in 1:3 resonance, but the Fourier amplitude at the second frequency is too large to be simply the second overtone of the fundamental frequency. The masses of the resulting third and fourth components indicate that they might be red or white dwarfs.

\subsection{Group III. (Unlikely Cases)}

In the case of the other ten stars we could not find any corresponding good third body orbit. Some of them were mentioned as candidates for triple (XZ And:

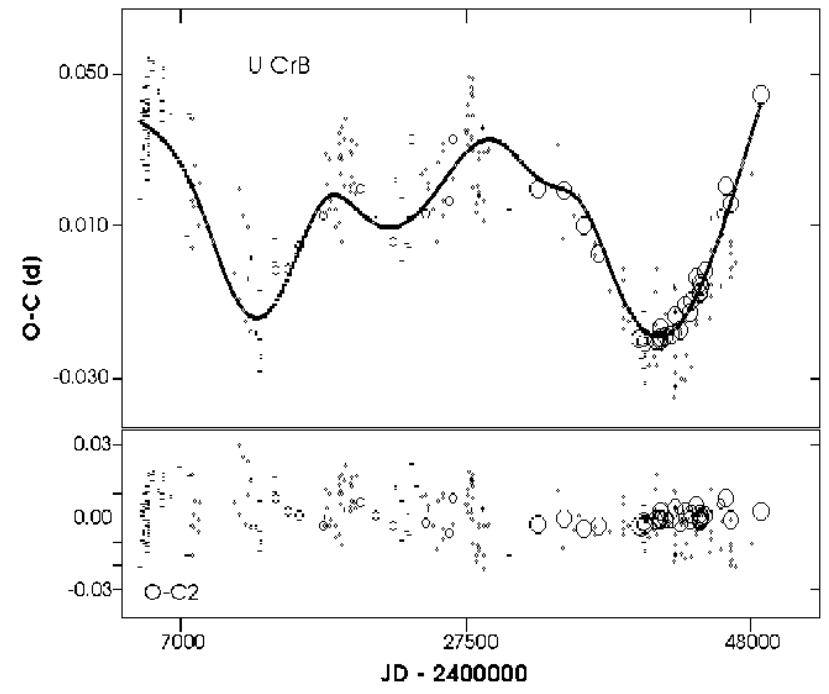

Fig. 16. A four body representation for the TOT points of $\mathrm{U} \mathrm{CrB}$

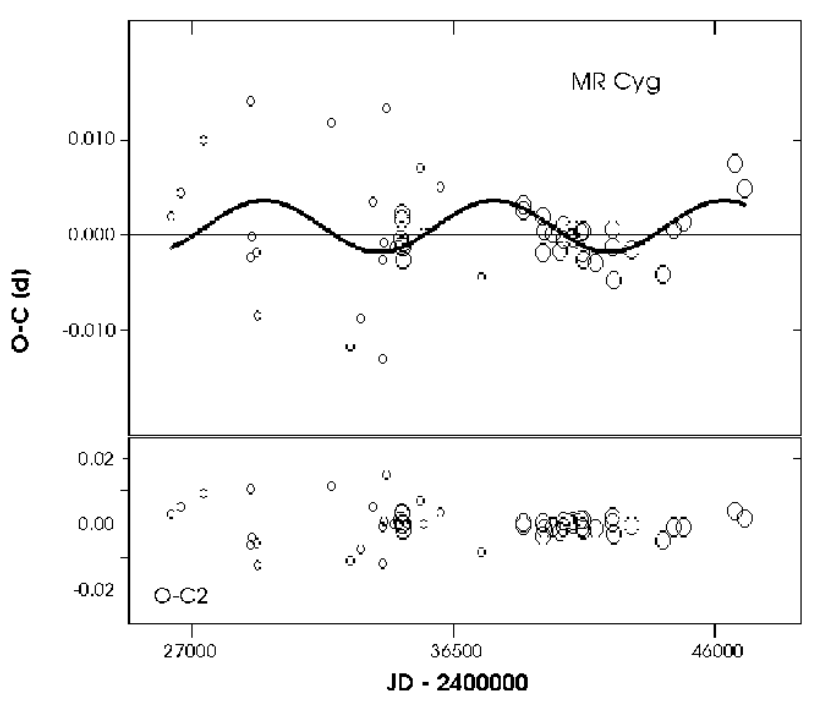

Fig. 17. PGPE points of MR Cyg with LITE solution made after the removal of a parabola

Frieboes-Conde \& Herczeg 1973; OO Aql: Demircan \& Güdür 1981; U CrB: Bakos \& Tremko 1981) or multiple (Y Cam: Mossakovskaya 1993; SW Lac: Panchatsaram \& Abhyankar 1982a) stellar systems in the earlier literature. We can neither agree with these previous results nor suggest any better explanations (e.g. Bakos \& Tremko's fit is excellent for the photoelectric times of minima of $\mathrm{U} \mathrm{CrB}$, but it differs significantly from the data at earlier epochs).

For the sake of completeness we present the computed third and/or fourth body orbits for eight stars out of this sample of ten. The elements can be found in Table 5, and the fits in Figs. 10-17. In the case of TW Cas and SW Lac we could not find any LITE solution at all. 
Table 3. Solutions for group I. systems. $P_{\text {orb }}^{\prime}$ (in days), $e^{\prime}$, $\omega^{\prime}$ (in $\left.\mathrm{rad}\right), \tau^{\prime}($ in $\mathrm{JD}-2400000), a^{\prime} \sin i^{\prime}\left(10^{6} \mathrm{~km}\right)$ are the orbital elements of the orbit of the eclipsing binary in the triple (multiple) system. $f\left(m_{3}\right)$ is the mass function of the third body (in solar mass), $m_{3}$ is the mass of the third (fourth) body at different inclinations (in solar mass). $\sigma_{\mathrm{Fr}}$ and $\sigma_{\mathrm{O}-\mathrm{C}}$ are the standard deviations of the data with respect to Fourier-fitting and the LITE orbit of above elements, respectively

\begin{tabular}{|c|c|c|c|c|}
\hline & AB And & TV Cas & XX Cep & AK Her \\
\hline Remarks: & $1,2,3$ & $1,2,3$ & 2,3 & 2,3 \\
\hline$P_{\text {orb }}^{\prime}$ & 19765 & 21412 & 21888 & 27243 \\
\hline$e^{\prime}$ & 0.14 & 0.16 & 0.26 & 0.33 \\
\hline$\omega^{\prime}$ & 1.30 & 0.53 & 1.32 & 5.10 \\
\hline$\tau^{\prime}$ & 22570 & 37774 & 31055 & 32442 \\
\hline$a^{\prime} \sin i^{\prime}$ & 413 & 220 & 968 & 382 \\
\hline$f\left(m_{3}\right)$ & 0.007 & 0.001 & 0.075 & 0.003 \\
\hline$m_{3} 90^{\circ}$ & 0.42 & 0.32 & 0.89 & 0.21 \\
\hline $60^{\circ}$ & 0.49 & 0.37 & 1.07 & 0.24 \\
\hline $30^{\circ}$ & 0.92 & 0.61 & 2.30 & 0.45 \\
\hline$\sigma_{\mathrm{Fr}}$ & 0.0022 & 0.0023 & 0.0026 & 0.0021 \\
\hline$\sigma_{\mathrm{O}-\mathrm{C}}$ & 0.0022 & 0.0023 & 0.0029 & 0.0021 \\
\hline
\end{tabular}

Remarks:

1: results obtained by subtracting quadratic ephemeris 2: PGPE type results (using only photoelectric and photographic minima)

3: final results obtained omitting the data outside the $3 \sigma$ belt.

\section{Conclusion}

We studied a group of stars for achieving modified or new interpretations of their $\mathrm{O}-\mathrm{C}$ diagrams. We could verify and/or improve the orbital elements of third or further satellites of some eclipsing systems studied earlier. In several cases our results are in contradiction with those of previous studies.

LITE is a very attractive explanation for wave-like $\mathrm{O}-\mathrm{C}$ behaviour. However, one must be very careful with the decision whether the results can be acceptable (physically reasonable) or not. In each case it is crucial to obtain new observational data in the future. We shall continue this investigation, by taking into account further eclipsing binaries, and also by monitoring the systems studied here for checking whether the forthcoming times of minima will follow our theoretical approximation or not. The use of independent observational methods would be advisable for these stars to confirm or discard the third body hypothesis.

Acknowledgements. The work was partly supported by the OTKA T4330 and OTKA F7318 National Grants, 'For the Hungarian Science' Foundation, and the Local Government of Bács-Kiskun County. We would like to express our great ac- knowledgement to late Dieter Lichtenknecker for sending his excellent data collection which has made our work much easier and faster. T.B. would like to acknowledge the technical support (computer accounts, telescope times and library work) for the Konkoly Observatory, Zs. Paragi and I.B. Bíró. Both of us appreciate to Drs. K. Szatmáry, J. Vinkó (JATE) and L. Szabados (Konkoly Obs.) for their advice. Last, but not least we would like to thank Prof. H. Drechsel (the referee) for his patience and useful suggestions.

\section{References}

Abhyankar K.D., Panchatsaram T., 1982, Bull. Astron. Soc. India 10, 315

Applegate J.H., Patterson J., 1987, ApJ 322, L99

Bakos G.A., Tremko J., 1981, J. R. Astron. Soc. Can. 75, 124

Barker L.A., Herczeg T.J., 1979, PASP 91, 247

Budding E., 1984, Bull. Inf. CDS 27, 91

Chambliss C.R., 1992, PASP 104, 663

de Loore C.W.H., Doom C., 1992, Structure and Evolution of Single and Binary Stars, Kluwer Acad. Publ., p. 6

Demircan O., Güdür N., 1981, Photometric and Spectroscopic Binary Systems. In: Carling and Kopal (eds.). D. Reidel Co., p. 413

Fresa F., 1956, Mem. Soc. Astron. Ital. 27, 150

Frieboes-Conde H., Herczeg T.J., 1973, A\&AS 12, 1

Giannone P., Giannuzzi M.A., 1974, Ap\&SS 26, 289

Hegedüs T., 1988, Bull. Inf. CDS 35, 15

Heintze J.R.W., 1990, NATO Adv. Stud. Inst. on Active Close Binaries. In: Ibanoglu (ed.). Kluwer Acad. Publ., p. 219

Hill G., Barnes J.V., 1972, PASP 84, 430

Hrivnak B.J., 1989, ApJ 340, 458

Kartasheva T.A., Svechnikov M.A., 1988, Zvezdi Typa WolfRayet i Rodstv. im. Obj., Mater. Vsesoj. Sovesc., Yelva, 14-17 Oct. 1986, Tallin, p. 126

Kholopov P.N., et al., 1985, General Catalog of Variable Stars, 4th Eds.

Kopal Z., 1978, Dynamics of Close Binary Systems. D. Reidel. Co.

Kreiner J.M., 1971, Acta Astron. 21, 365

Lichtenknecker D., 1988, (personal communications)

Linnell A.P., Kallrath J., 1987, ApJ 316, 754

Mayer P., 1984, Bull. Astron. Inst. Czech. 35, 180

Mayer P., 1990, Bull. Astron. Inst. Czech. 41, 231

Mayer P., Wolf M., Tremko J., Niarchos P.G., 1991, Bull. Astron. Inst. Czech. 42, 225

Mayor M., Mazeh T., 1987, A\&A 171, 157

Mossakovskaya L.V., 1993, IBVS 3902

Nagy T.A., 1985, PASP 97, 1005

Panchatsaram T., Abhyankar K.D., 1982a, Binary and Multiple Stars as Tracers of Stellar Evolution. In: Kopal, Rache (eds.), p. 47

Panchatsaram T., Abhyankar K.D., 1982b, Bull. Astron. Soc. India 10, 111

Plavec M., 1959, Bull. Astron. Inst. Czech. 10, 185

Plavec M., Smetanová M, Pekny Z., 1961, Bull. Astron. Inst. Czech. 12, 117

Rafert J.B., 1982, PASP 94, 485 
Table 4. Designations as in Table 3

\begin{tabular}{|c|c|c|c|c|c|c|}
\hline \multirow{3}{*}{ Remarks: } & \multicolumn{2}{|c|}{ W Del } & \multirow{3}{*}{$\frac{\text { U Peg }}{3}$} & \multirow{3}{*}{$\begin{array}{l}\text { AT Peg } \\
1,3,4\end{array}$} & \multicolumn{2}{|c|}{ ST Per } \\
\hline & \multicolumn{2}{|c|}{1,2} & & & \multicolumn{2}{|c|}{2} \\
\hline & $3 \mathrm{rd} *$ & 4 th $*$ & & & $3 \mathrm{rd} *$ & $4 \operatorname{th} *$ \\
\hline$P_{\text {orb }}^{\prime}$ & 21444 & 13433 & 49721 & 9169 & 27000 & 9412 \\
\hline$e^{\prime}$ & 0.36 & 0.18 & 0.43 & 0.39 & 0.19 & 0.24 \\
\hline$\omega^{\prime}$ & 4.44 & 4.30 & 3.55 & 6.05 & 5.80 & 6.36 \\
\hline$\tau^{\prime}$ & 29106 & 38836 & 50631 & 41011 & 48543 & 39904 \\
\hline$a^{\prime} \sin i^{\prime}$ & 1095 & 568 & 1189 & 291 & 679 & 427 \\
\hline$f\left(m_{3}\right)$ & 0.113 & 0.040 & 0.027 & 0.012 & 0.017 & 0.035 \\
\hline$m_{3} 90^{\circ}$ & 1.12 & 0.74 & 0.59 & 0.54 & 0.53 & 0.70 \\
\hline $60^{\circ}$ & 1.36 & 0.88 & 0.69 & 0.63 & 0.62 & 0.80 \\
\hline $30^{\circ}$ & 2.98 & 1.79 & 1.39 & 1.20 & 1.22 & 1.67 \\
\hline$\sigma_{\mathrm{Fr}}$ & \multicolumn{2}{|c|}{0.0114} & 0.0021 & 0.0013 & \multicolumn{2}{|c|}{0.0062} \\
\hline$\sigma_{\mathrm{O}-\mathrm{C}}$ & \multicolumn{2}{|c|}{0.0125} & 0.0022 & 0.0021 & \multicolumn{2}{|c|}{0.0062} \\
\hline
\end{tabular}

Remarks:

1: results obtained by subtracting quadratic ephemeris

2: TOT type results (using all kind of minima times)

3: PGPE type results (using only photoelectric and photographic minima)

4: final results contain the exclusion of the data out of the $3 \sigma$.

Table 5. Designations as in Table 3

\begin{tabular}{|c|c|c|c|c|c|c|c|c|c|c|c|}
\hline \multirow{3}{*}{ Remarks: } & \multicolumn{2}{|c|}{ RT And } & \multicolumn{2}{|c|}{$\mathrm{XZ}$ And } & \multirow{3}{*}{$\begin{array}{l}\text { OO Aql } \\
1,2\end{array}$} & \multirow{3}{*}{$\frac{\text { Y Cam }}{2}$} & \multirow{3}{*}{$\begin{array}{l}\text { RS CVn } \\
1,3\end{array}$} & \multirow{3}{*}{$\begin{array}{l}\text { CQ Cep } \\
1,2\end{array}$} & \multicolumn{2}{|c|}{$\mathrm{U}$ CrB } & MR Cyg \\
\hline & \multicolumn{2}{|c|}{3} & \multicolumn{2}{|c|}{2} & & & & & \multicolumn{2}{|c|}{2} & 1,4 \\
\hline & $3 \mathrm{rd} *$ & 4 th $*$ & $3 \mathrm{rd} *$ & 4 th $*$ & & & & & $3 \mathrm{rd} *$ & 4 th $*$ & \\
\hline$P_{\mathrm{orb}}^{\prime}$ & 40373 & 18680 & 25500 & 13000 & 9214 & 50408 & 21809 & 19974 & 21955 & 18680 & 8343 \\
\hline$e^{\prime}$ & 0.56 & 0.51 & 0.15 & 0.33 & 0.22 & 0.35 & 0.54 & 0 & 0.24 & 0.30 & 0 \\
\hline$\omega^{\prime}$ & 4.38 & 0.03 & 3.77 & 1.47 & 2.57 & 1.04 & 0.42 & 0 & 2.69 & 1.67 & 0 \\
\hline$\tau^{\prime}$ & 20202 & 28718 & 7347 & 15205 & 39897 & 62638 & 5519 & 19359 & 30959 & 35900 & 27544 \\
\hline$a^{\prime} \sin i^{\prime}$ & 769 & 61 & 874 & 496 & 126 & 13750 & 2036 & 422 & 1009 & 788 & 71 \\
\hline$f\left(m_{3}\right)$ & 0.011 & 0.00003 & 0.039 & 0.027 & 0.001 & 40.013 & 0.703 & 0.008 & 0.085 & 0.057 & 0.0002 \\
\hline$m_{3} 90^{\circ}$ & 0.46 & 0.05 & 1.09 & 0.94 & 0.19 & 45.18 & 2.79 & 2.34 & 1.73 & 1.48 & 0.34 \\
\hline $60^{\circ}$ & 0.54 & 0.06 & 1.29 & 1.11 & 0.22 & 66.92 & 3.49 & 2.71 & 2.05 & 1.75 & 0.39 \\
\hline $30^{\circ}$ & 1.04 & 0.11 & 2.53 & 2.15 & 0.40 & 325.69 & 9.42 & 4.86 & 4.14 & 3.46 & 0.69 \\
\hline$\sigma_{\mathrm{Fr}}$ & \multirow{2}{*}{\multicolumn{2}{|c|}{0.0019}} & \multirow{2}{*}{\multicolumn{2}{|c|}{0.0063}} & 0.0045 & & 0.0157 & \multicolumn{4}{|c|}{0.0070} \\
\hline$\sigma_{\mathrm{O}-\mathrm{C}}$ & & & & & 0.0048 & 0.0147 & 0.0201 & 0.0123 & & & 0.052 \\
\hline
\end{tabular}

Remarks:

1: results obtained by subtracting quadratic ephemeris

2: TOT type results (using all kind of minima times)

3: PGPE type results (using only photoelectric and photographic minima)

4: PE type results (using only photoelectric minima).

Rovithis-Livaniou H., Niarchos P.G., Rovithis P., 1990, NATO Adv. Stud. Inst. on Active Close Binaries. In: Ibanoglu (ed.). Kluwer Acad. Publ., p. 253

Russell H.N., Fowler M., Borton M., 1917, ApJ 45, 306 van Hamme W., Wilson R.E., 1990, AJ 100, 1981

Vinkó J., 1989, Diploma-Thesis, JATE University, Szeged, Hungary

Woodward E.J., Wilson R.E., 1977, Ap\&SS 52, 387 